\title{
Aharonov-Bohm Effect for Parallel and T-shaped Double Quantum Dots
}

\author{
Yoichi TANAKA and Norio KAWAKAMI \\ Department of Applied Physics, Osaka University, Suita, Osaka 565-0871
}

(Received January 30, 2019)

\begin{abstract}
We investigate the Aharonov-Bohm (AB) effect for the double quantum dots in the Kondo regime using the slave-boson mean-field approximation. In contrast to the non-interacting case, where the $\mathrm{AB}$ oscillation generally has the period of $4 \pi$ when the two-subring structure is formed via the interdot tunneling $t_{c}$, we find that the $\mathrm{AB}$ oscillation has the period of $2 \pi$ in the Kondo regime. Such effects appear for the double quantum dots close to the T-shaped geometry even in the charge-fluctuation regime. These results follow from the fact that the Kondo resonance is always fixed to the Fermi level irrespective of the detailed structure of the bare dot-levels.
\end{abstract}

KEYWORDS: Aharonov-Bohm effect, Kondo effect, Double Quantum Dots

Recent rapid progress in the nanotechnology makes it possible to fabricate double quantum dot (DQD) systems. ${ }^{1}$ One of the remarkable features in the DQD systems is the interference effect induced via multiple paths of electron propagation, which is very sensitive to the external magnetic flux, giving rise to the AharonovBohm (AB) effect. ${ }^{2,3}$ The $\mathrm{AB}$ effect has been theoretically studied for the DQD with ${ }^{4-8}$ or without ${ }^{9-13}$ the intradot Coulomb interaction. As noted in the literature recently, ${ }^{10-13}$ the interdot tunneling between two dots, which forms two-subring structure, generates a new $\mathrm{AB}$ oscillation period of $4 \pi$ instead of the normal period of $2 \pi$. However, the arguments are based on the noninteracting electron model, and the influence of electron correlations on this new $\mathrm{AB}$ oscillation has not been discussed yet.

In this note, we address this question about the $A B$ effect by explicitly taking into account the Kondo effect in the DQD shown in Fig. 1. We will show that if the

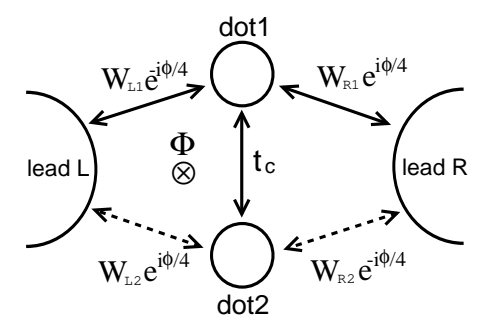

Fig. 1. DQD system with the magnetic flux $\Phi$.

Kondo effect is taken into account in the parallel $\mathrm{DQD}$, the $\mathrm{AB}$ period should be practically regarded as $2 \pi$ instead of $4 \pi$. This tendency becomes more remarkable in the DQD close to the T-shaped geometry, which is another typical DQD system sensitive to the interference effect.

In the following discussions, the intradot Coulomb interaction is assumed to be sufficiently large, so that double occupancy at each QD is forbidden. This assumption which allows us to use a slave-boson representation of correlated electrons in the dots. In this representation, the DQD system in Fig. 1 is modeled with the $N(=2)$ fold degenerate Anderson Hamiltonian

$$
\begin{aligned}
H & =\sum_{k_{\alpha}, \sigma} \varepsilon_{k_{\alpha}} c_{k_{\alpha} \sigma}^{\dagger} c_{k_{\alpha} \sigma}+\sum_{m, \sigma} \varepsilon_{d} f_{m \sigma}^{\dagger} f_{m \sigma} \\
& +\frac{t_{c}}{N} \sum_{\sigma}\left(f_{1 \sigma}^{\dagger} b_{1} b_{2}^{\dagger} f_{2 \sigma}+h . c\right) \\
& +\frac{1}{\sqrt{N}} \sum_{k_{\alpha}, m, \sigma}\left(V_{\alpha m} c_{k_{\alpha} \sigma}^{\dagger} b_{m}^{\dagger} f_{m \sigma}+\text { h.c. }\right)
\end{aligned}
$$

where $c_{k_{\alpha} \sigma}$ is the annihilation operator of an electron with spin $\sigma$ in the lead $\alpha(\alpha=L, R)$, and the annihilation operator of an electron in the dot $m(m=$ $1,2)$ with the energy $\varepsilon_{d}$ is represented as $b_{m}^{\dagger} f_{m \sigma}$ with the constraint, $f_{m \sigma}^{\dagger} f_{m \sigma}+b_{m}^{\dagger} b_{m}=1$, where $b_{m}\left(f_{m \sigma}\right)$ is the slave-boson (pseudo-fermion) annihilation operator for an empty state (singly occupied state). We use the mean-field approximation at zero temperature (see ref.14). The effect of the magnetic flux $\Phi$ is symmetrically incorporated in the tunneling between the dot $m$ and the lead $\alpha$ in eq. (1), $V_{L 1(R 2)}=W_{L 1(R 2)} e^{-i \phi / 4}$, $V_{L 2(R 1)}=W_{L 2(R 1)} e^{i \phi / 4}$, where $\phi=2 \pi \Phi / \Phi_{0}\left(\Phi_{0}=h / e\right)$. This means that the magnetic flux equally pierces the two subrings formed by the interdot tunneling $t_{c}$. We systematically change the arrangement of the DQD by modifying the resonance width $\Gamma_{1,1(2,2)}^{\alpha}=\pi\left|W_{\alpha 1(2)}\right|^{2} \rho(\rho$ is the density of states for leads at the Fermi energy): the parallel DQD is given by $\Gamma_{1,1}^{\alpha} \sim \Gamma_{2,2}^{\alpha}$, and the T-shaped DQD by $\Gamma_{2,2}^{\alpha} \sim 0$ with fixed $\Gamma_{1,1}^{\alpha} \cdot{ }^{15}$

Figure 2 shows the conductance as a function of the magnetic flux $\phi$ for several values of $\Gamma_{2,2}^{\alpha}$. We have studied two typical cases in the Kondo regime and the chargefluctuation regime. There are several remarkable properties in the conductance. (i) As seen in Fig. 2(a), the AB period of the conductance seems to be always $2 \pi$ in the Kondo regime $\left(\varepsilon_{d}=-3.5\right)$ even if we change the arrangement of the DQD from the parallel to T-shaped geometry. This is in contrast to the conclusion for the noninteracting case: the period should be $4 \pi$ generally, ${ }^{10-13}$ which follows from the two-subring structure formed via the interdot tunneling $t_{c}$. (ii) On the other hand, in the charge-fluctuation regime $\left(\varepsilon_{d}=-2.4\right)$ in Fig. 2(b), the $\mathrm{AB}$ oscillation for the parallel DQD $\left(\Gamma_{2,2}^{\alpha}=0.9\right)$ indeed 


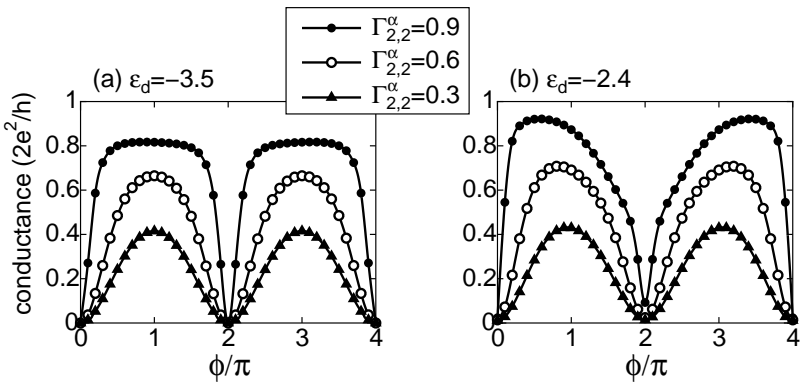

Fig. 2. Conductance as a function of the magnetic flux $\phi$ : (a) Kondo regime, (b) charge-fluctuation regime. We choose the interdot coupling $t_{c}=1.5$ in the unit of $\Gamma_{1,1}^{\alpha}(=1)$.

has the period of $4 \pi$. Even in this case, however, as the DQD system is changed from the parallel to T-shaped geometry, the period again approaches $2 \pi$ (see $\Gamma_{2,2}^{\alpha}=0.3$ in Fig. 2(b)). These results imply that electron correlations play a crucial role for the $\mathrm{AB}$ oscillation in the $\mathrm{DQD}$.

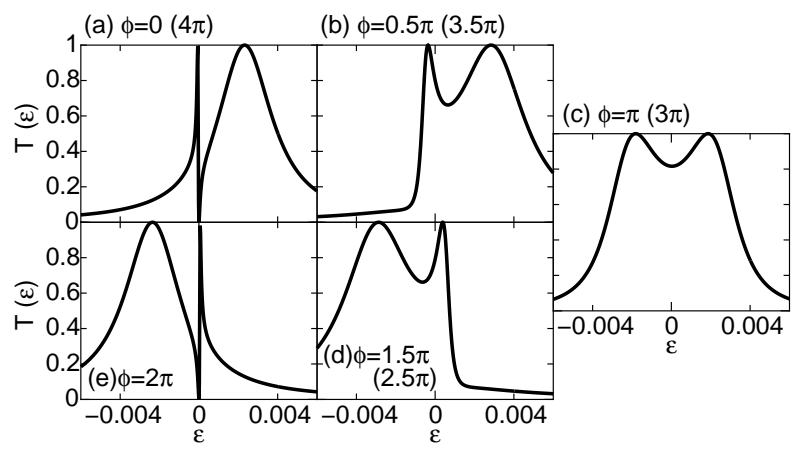

Fig. 3. Transmission probability $T(\varepsilon)$ around the Fermi energy $(\varepsilon=0)$ in the Kondo regime $\left(\varepsilon_{d}=-3.5\right)$. We set $t_{c}=1.5$ and $\Gamma_{2,2}^{\alpha}=0.9$.

The above characteristic properties result from the Kondo resonances affected by the interference effect. In Fig. 3 the transmission probability $T(\varepsilon)$ is shown around the Fermi energy $(\varepsilon=0)$ in the case close to the parallel geometry $\left(\Gamma_{2,2}^{\alpha}=0.9\right)$. At $\phi=0$, the Kondo resonance splits into two distinct resonances due to the interdot coupling $t_{c}$ : they consist of a sharp bonding Kondo resonance at the Fermi energy and a broad anti-bonding Kondo resonance. ${ }^{14}$ The interference between these two resonances produces a dip in $T(\varepsilon)$ around $\varepsilon=0$, as seen in Fig. 3(a). As the magnetic flux $\phi$ increases, the interference effect is smeared (Fig. 3(c)), and the Kondo resonances in $T(\varepsilon)$ become almost symmetric at $\phi=\pi$. At $\phi=2 \pi$, the shape of $T(\varepsilon)$ is inverted with respect to the Fermi energy as shown in Fig. 3(e), and then $T(\varepsilon)$ returns to the original shape with another increase by $2 \pi$. We thus see that $T(\varepsilon)$ changes with the period of $4 \pi$, as should be expected. However, it should be noted that the value of $T(\varepsilon=0)$ oscillates with the period very close to $2 \pi$, which characterizes the linear conductance shown in Fig. 2(a) for $\Gamma_{2,2}^{\alpha}=0.9$. This new periodicity is due to the constraint imposed on the Kondo resonances: the self-energy shift inherent in the Kondo effect properly tunes the position of the resonances so as to keep the electron number in the dots constant. This many-body effect gives rise to the effective period of $2 \pi$, in contrast to the non-interacting case. ${ }^{10-13}$

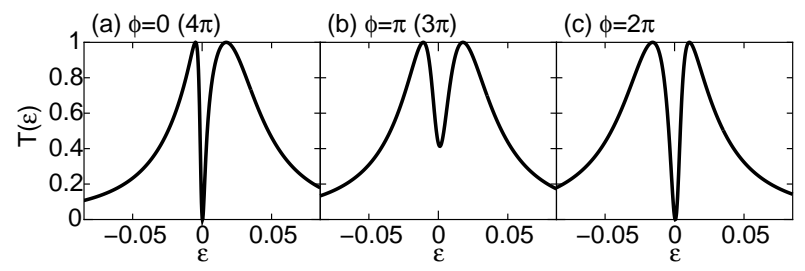

Fig. 4. Transmission Probability $T(\varepsilon)$ for $\Gamma_{2,2}^{\alpha}=0.3$ in the charge-fluctuation regime $\left(\varepsilon_{d}=-2.4\right)$.

In the charge-fluctuation regime $\left(\varepsilon_{d}=-2.4\right)$, the resonances are both lifted slightly above the Fermi energy. The resulting asymmetric nature in $T(\varepsilon)$ naturally gives the $\mathrm{AB}$ effect with the period of $4 \pi$, as seen for $\Gamma_{2,2}^{\alpha}=0.9$ in Fig. 2(b). However, even in this charge-fluctuation regime, we encounter a remarkable fact that when the system approaches the T-shaped DQD $\left(\Gamma_{2,2}^{\alpha}=0.3\right.$ in Fig. $2(\mathrm{~b})$ ), the effective period becomes $2 \pi$ again. To understand this tendency, we should recall that while the whole DQD system is in the charge-fluctuation regime, the dot2 itself falls into the Kondo regime because it is almost decoupled from the leads in the T-shaped geometry. We can see that the broad resonance of the dot-1 develops a dip structure at the Fermi energy, which is caused by the sharp Kondo resonance formed in the dot-2. In this case, the DOS of the dot-1 mainly controls the transmission probability $T(\varepsilon)$ shown in Fig. 4 . When the magnetic flux $\phi$ is turned on, the dip in $T(\varepsilon)$ is slightly modified with the resonance-width almost unchanged. Therefore, although the current flows mainly through the dot- 1 in the T-shaped geometry, the sharp dip structure caused by the Kondo effect in the dot-2 essentially controls the linear conductance in the system. This gives rise to $2 \pi$ for the effective $\mathrm{AB}$ period even in the charge-fluctuation regime, as observed in Fig. 2(b).

Finally, we would like to comment on the $\mathrm{AB}$ period in more general cases for which the magnetic flux piercing the two subrings is not equal: the ratio of flux is assumed to be an irreducible fraction $m / n$. In the non-interacting case, the $\mathrm{AB}$ period of the conductance is $2 \pi(m+n)$. In the Kondo regime, however, the $\mathrm{AB}$ period becomes half, $\pi(m+n)$, provided that both $m$ and $n$ are odd, while it remains $2 \pi(m+n)$ if either $m$ or $n$ is even.

In summary, we have shown that in spite of the twosubring structure in our DQD system, the AB oscillation practically has the period of $2 \pi$ in the Kondo regime. Such effects appear for the DQD close to the Tshaped geometry even in the charge-fluctuation regime. This conclusion, which is contrasted to the noninteracting case, ${ }^{10-13}$ arises from the self-energy shift inherent in the Kondo effect: the Kondo resonance is always fixed to the Fermi level irrespective of the detailed structure of the bare dot-levels. 
1) For a review see e.g. W. G. van der Wiel, S. De Franceschi, J. M. Elzerman, T. Fujisawa, S. Tarucha and L. P. Kouwenhoven: Rev. Mod. Phys. 75 (2003) 1.

2) A. W. Holleitner, C. R. Decker, H. Qin, K. Eberl and R. H. Blick: Phys. Rev. Lett. 87 (2001) 256802.

3) M. Sigrist, A. Fuhrer, T. Ihn, K. Ensslin, S. E. Ulloa, W.Wegscheider and M. Bichler: Phys. Rev. Lett. 93 (2004) 066802 .

4) H. Akera: Phys. Rev. B 47 (1993) 6835.

5) W. Izumida, O. Sakai and Y. Shimizu: J. Phys. Soc. Jpn. 66 (1997) 717 .

6) D. Boese, W. Hofstetter and H. Schoeller: Phys. Rev. B 66 (2002) 125315.

7) Y. Utsumi, J. Martinek, P. Bruno and H. Imamura: Phys. Rev. B 69 (2004) 155320.
8) R. López, D. Sánchez, M. Lee, M. -S. Choi, P. Simon and K. L. Hur: Phys. Rev. B 71 (2005) 115312.

9) B. Kubala and J. König: Phys. Rev. B 65 (2002) 245301.

10) Z. T. Jiang, J. Q. You, S. B. Bian and H. Z. Zheng: Phys. Rev. B 66 (2002) 205306.

11) P. A. Orellana, M. L. Ladrón de Guevara and F. Claro: Phys. Rev. B 70 (2004) 233315.

12) K. Kang and S. Y. Cho: J. Phys.: Condens. Matter 16 (2004) 117.

13) Z. -M. Bai, M. -F. Yang and Y. -C. Chen: J. Phys.: Condens. Matter 16 (2004) 2053.

14) Y. Tanaka and N. Kawakami: J. Phys. Soc. Jpn. 73 (2004) 2795; Phys. Rev. B 72 (2005) 085304.

15) Y. Takazawa, Y. Imai, and N. Kawakami: J. Phys. Soc. Jpn. 71 (2002) 2234 